\title{
The microbial community shifts of subgingival plaque in patients with generalized aggressive periodontitis following non-surgical periodontal therapy: a pilot study
}

\author{
Jing Han ${ }^{1,2}$, Peng Wang ${ }^{1,2}$ and Shaohua $\mathbf{G e}^{1,2}$ \\ ${ }^{1}$ Shandong Provincial Key Laboratory of Oral Tissue Regeneration, School of Stomatology, Shandong University, Jinan, China \\ 2 Department of Periodontology, School of Stomatology, Shandong University, Jinan, China \\ Correspondence to: Shaohua Ge, email: shaohuage@sdu.edu.cn \\ Keywords: generalized aggressive periodontitis, metagenome, subgingival plaque, non-surgical treatment, bacterial community \\ shifts \\ Received: August 15, $2016 \quad$ Accepted: September 29, $2016 \quad$ Published: October 08, 2016
}

\section{ABSTRACT}

The object of this study is to characterize the bacterial community of subgingival plaque of two subjects with generalized aggressive periodontitis (GAgP) pre- and posttreatment. We picked two patients with GAgP and used high-throughput 16S rDNA sequencing. V4 hypervariable region was picked for PCR amplification of subgingival samples. Then, the PCR products were sequenced through Illumina MiSeq platform. One month after therapy, both the clinical features and periodontal parameters improved obviously. Moreover, the composition and structure of subgingival bacterial community changed after initial periodontal therapy. Also, the composition of the subgingival microbiota was highly individualized among different patients. Bacteroidetes, Spirochaetes and Fusobacteria were related to pathogenicity of GAgP while Actinobacteria and Proteobacteria seemed associated with clinical symptoms resolution. In this study, we found the subgingival bacterial community was high in species richness but dominated by a few species or phylotypes, with significant shifts of microbiota that occurred after treatment. This study demonstrated the shift of the subgingival bacterial community before and after treatment by high-throughput $16 \mathrm{~S}$ rDNA sequencing, and provided a concise method for analysis of microbial community for periodontal diseases.

\section{INTRODUCTION}

The oral cavity contains a large number of ecological sites that provide surfaces for microbial colonization. Studies revealed that the predominant bacteria were normal flora, which were non-pathogenic and helped prevent colonization by exogenous organism, and were considered to be commensal in the oral cavity [1, 2]. However, some of the bacteria are associated with oral diseases, such as dental caries and periodontal diseases. By culture method, approximately 200 bacterial species have been found in the oral cavity. Using a metagenomic approach first proposed by Kroes et al [3] and Paster et al [4], over 1000 phylotypes have been detected in the oral samples [5], which raised the possibility that uncultivated and as-yet-uncharacterized species might also participate in the etiology of oral diseases [6]. It is now well recognized that periodontitis is a kind of polymicrobial infection. However, only about half of the subgingival bacterial species or phylotypes are cultivable, which presents an obstacle to fully understand the causal relationship between subgingival bacteria and periodontitis [7]. Most of our understanding of subgingival bacteria comes from cultivable bacteria, and the role of many noncultivable bacteria remains largely unknown. Furthermore, whether the microbial community shifts before and after periodontal therapy remains unclear. Metagenomic study involves the identification, sequencing, and functional and transcriptome analysis of environmental samples. Using metagenomic methods, the members of the microbial community are typically determined by tracking phylogenetic markers such as the 16S rDNA.

$\mathrm{GAgP}$ represents a specific type of periodontitis with clearly identifiable clinical and laboratory findings that are different from other forms of periodontitis. 
GAgP is characterized by the relatively young ages of the affected individual (usually 30 years old or older), and a generalized loss of attachment and alveolar bone except in incisors, first molars and other permanent teeth (besides at least three permanent teeth) [8]. Actually, GAgP usually affected most teeth of oral cavity. It has been revealed that the predominant bacteria of GAgP include Actinobacillus actinomycetemcomitans (Aa), Porphyromonas gingivalis (Pg), Tannerella forsythensis (Tf), Treponema denticola (Td), Campylobacter rectus (Cr), Prevotella intermedia (Pi) and Prevotella nigrescens (Pn) $[9,10]$. However, the microbial community shifts pre- and post-treatment of GAgP is still unclear.

Therefore, our present study aimed to elucidate explorative and descriptive shifts in bacterial communities by next generation sequencing of subgingival plaque samples from two GAgP patients before and after mechanical debridement. The hypervariable region V4 of the $16 \mathrm{~S}$ rDNA gene was targeted to explore richness and diversity of subgingival plaque samples [11].

\section{RESULTS}

In this study, we analyzed subgingival plaque samples of two patients with GAgP for shifts in the microbial community in response to mechanical debridement. Demographic data, baseline and post intervention clinical parameters probing depth (PD) and bleeding on probing (BOP) were listed in Table S1. All clinical parameters improved after treatment. The intraoral photos and panoramic radiographs of two patients before and after treatment were shown in Figure S2 and Figure S3.

\section{Filtration and quality evaluation of original data}

A total of 952,272 V4 16s rDNA paired-end reads were obtained from the 16 samples (the minimum and maximum numbers of reads from the 16 samples were 12,564 and 100,089, respectively) (Figure 1). All of the 16 samples were used in this analysis. The raw reads were filtrated by QIIME quality filters. After filtrating, 915,626 sequence reads were left. The average length of the filtered sequence reads was $253 \mathrm{bp}$ (Table S2).

\section{Operational taxonomic unit-based analysis}

The taxon abundance of all 16 samples was generated into 34 phyla, 82 classes, 127 orders, 156 families and 187 genera. First, all operational taxonomic units (OTUs) picked from the 16 samples were normalized by homogenization procedure. Then, they were contrasted and hierarchical clustered intragroup mainly by using RDP classifier. Finally, we got Vene diagrams on this basis (Figure 2). The OTUs in each sample and the number of sequences in each OTU were counted to obtain the taxonomic information of the OTU. The percentage of shared OTUs among groups after treatment decreased. In contrast, the percentages of particular OTUs increased (Table S3). We supposed that prevalent microorganisms of GAgP suppressed the growth of other microorganisms

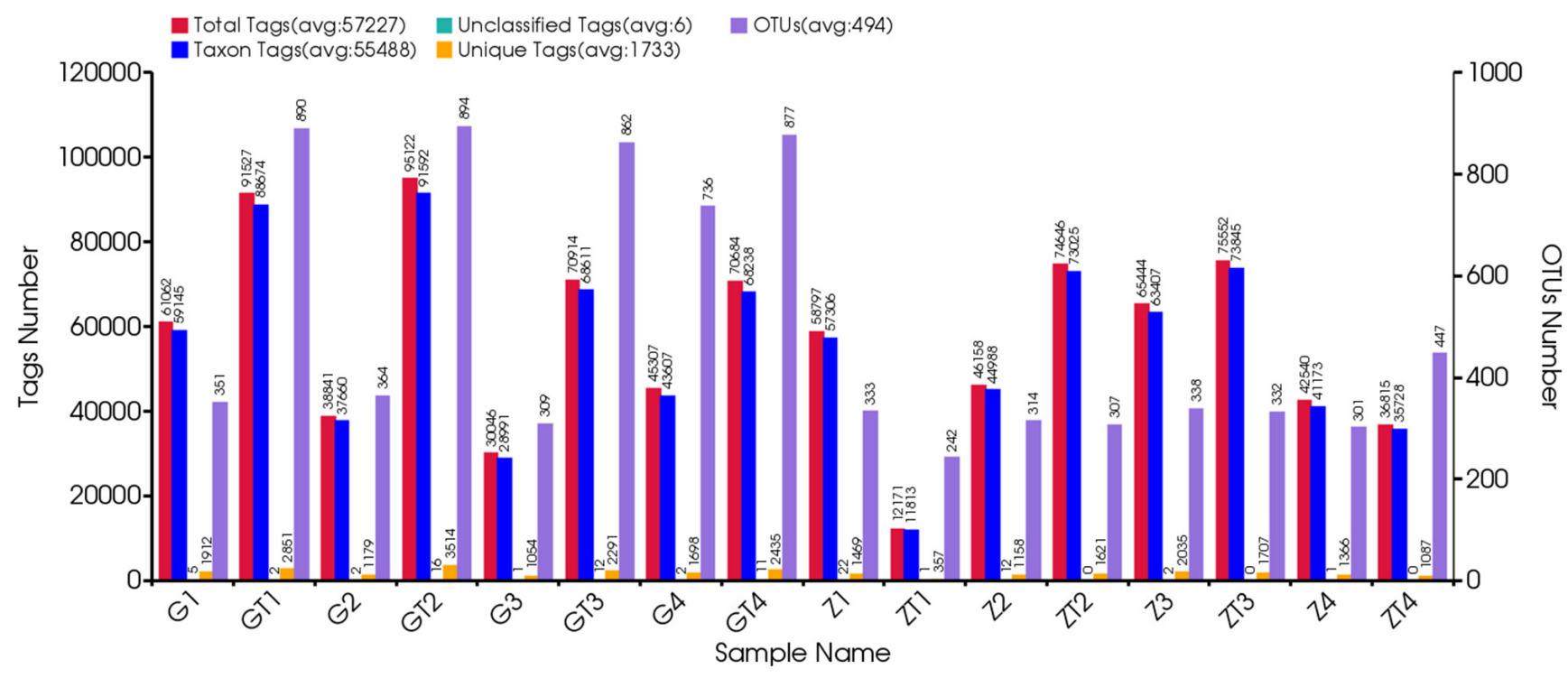

Figure 1: Statistical information of original data. The first vertical axis (Tags numbers):Total Tags means the number of merged tags after filtering; Taxon Tags means the number of tags which were used for establishing OTU table and obtained taxonomic information; Unclassified Tags means the number of tags which were used for establishing OTU table but did not obtain taxonomic information; Unique Tags means the number of tags whose frequency was 1 and can't be assigned to any OTUs. The second vertical axis (OTUs Number) represents the final OTU number after taxonomic analysis. 
Table 1: Comparison of the estimated operational taxonomic unit (OTU) richness, diversity index and Simpson index of $16 \mathrm{~S}$ rDNA gene libraries for clustering at $97 \%$ identity as obtained from the pyrosequencing analysis.

\begin{tabular}{|c|c|c|c|c|}
\hline Samples & Sequences number & Shannon & Chao1 & Simpson index \\
\hline G1 & 1190.0 & 6.00 & 212.3 & 0.75 \\
\hline G2 & 1190.0 & 6.23 & 220.0 & 0.75 \\
\hline G3 & 1190.0 & 6.25 & 219.5 & 0.76 \\
\hline G4 & 1190.0 & 6.77 & 352.2 & 0.78 \\
\hline GT1 & 1190.0 & 5.63 & 323.5 & 0.68 \\
\hline GT2 & 1190.0 & 6.14 & 349.8 & 0.77 \\
\hline GT3 & 1190.0 & 5.81 & 334.6 & 0.75 \\
\hline GT4 & 1190.0 & 6.09 & 370.5 & 0.75 \\
\hline Z1 & 1190.0 & 5.18 & 184.0 & 0.78 \\
\hline Z2 & 1190.0 & 4.86 & 171.7 & 0.78 \\
\hline Z3 & 1190.0 & 5.74 & 190.2 & 0.73 \\
\hline Z4 & 1190.0 & 4.98 & 170.7 & 0.75 \\
\hline ZT1 & 1190.0 & 3.99 & 154.6 & 0.69 \\
\hline ZT2 & 1190.0 & 3.32 & 117.7 & 0.66 \\
\hline ZT3 & 1190.0 & 2.42 & 147.3 & 0.42 \\
\hline ZT4 & 1190.0 & 2.17 & 204.1 & 0.30 \\
\hline
\end{tabular}

$\mathrm{G}$ and $\mathrm{Z}$ represented the samples from the patient $\mathrm{G}$ and $\mathrm{Z}$ before treatment; GT and $\mathrm{ZT}$ represented the samples from the patient $\mathrm{G}$ and $\mathrm{Z}$ after treatment.

which led to an increasing percentage of pathogenic microorganisms in microflora before treatment. After periodontal debridement, the growth of pathogenic microorganisms was suppressed, and the proportion of the pathogenic microorganisms decreased accordingly.

\section{Alpha diversity analysis}

Alpha diversity estimates the diversity in a specific area or ecosystem in terms of species richness which is estimated by rarefaction curves. The shape of the rarefaction curves indicates new phylotypes which will be expected with additional sequencing. The Shannon and Chao1 index curves of all samples reached plateaus with the current sequencing (Figure 3A, 3B), suggesting that most richness and diversity had already been captured. The subgingival plaques richness index was calculated as shown in Table 1. The Shannon index of diversity reflects both diversity and community evenness, and the Chaol index is an estimator of phylotype richness. Lower Shannon index after treatment indicated that the treatment reduced bacterial diversity within the subgingival plaque. Higher Chaol index after treatment indicated that treatment increased the bacterial richness of the subgingival plaque. These suggested that the group after treatment had a higher level of biodiversity and unevenness estimations than that before treatment. The result was confirmed by the Simpson Index (Table 1).

\section{Beta diversity analysis}

To further explore the relationship between different bacterial communities pre- and post-treatment, a PCoA analysis was performed using genus-level taxonomic profiles. UniFrac PCoA of 7897 (average 494) OTUs (grouped at $97 \%$ sequence identity) showed a clear separation between the samples pre and posttreatment (Figure 4A). Percentage values at the axes indicate contribution of the principal components to the explanation of total variance in the dataset. Figure 4A showed that the percentage of variation explained by PC1 and PC2 were $63.78 \%$ and $20.20 \%$. Samples before treatment formed a group apart from the two groups after treatment, which indicated that there was significant difference in the subgingival bacterial composition before and after treatment. The samples of two patients before treatment aggregated in the same group while distracted after treatment, which suggested that the bacterial composition of the two patients was similar before treatment, but exhibited significant difference after treatment. Interestingly, one sample (G4) before treatment was different, and not belonging to any of the three groups. The samples in the group before treatment were well separated from those in the group after treatment based on the Weighted UniFrac distance measured at the OTU level. The phylogenetic tree based on the Weighted UniFrac also revealed the separation between samples pre- and posttreatment, which was in accordance with PCoA result (Figure 4B). Based on the distance between weighted and unweighted UniFrac measured at the OTU level, 

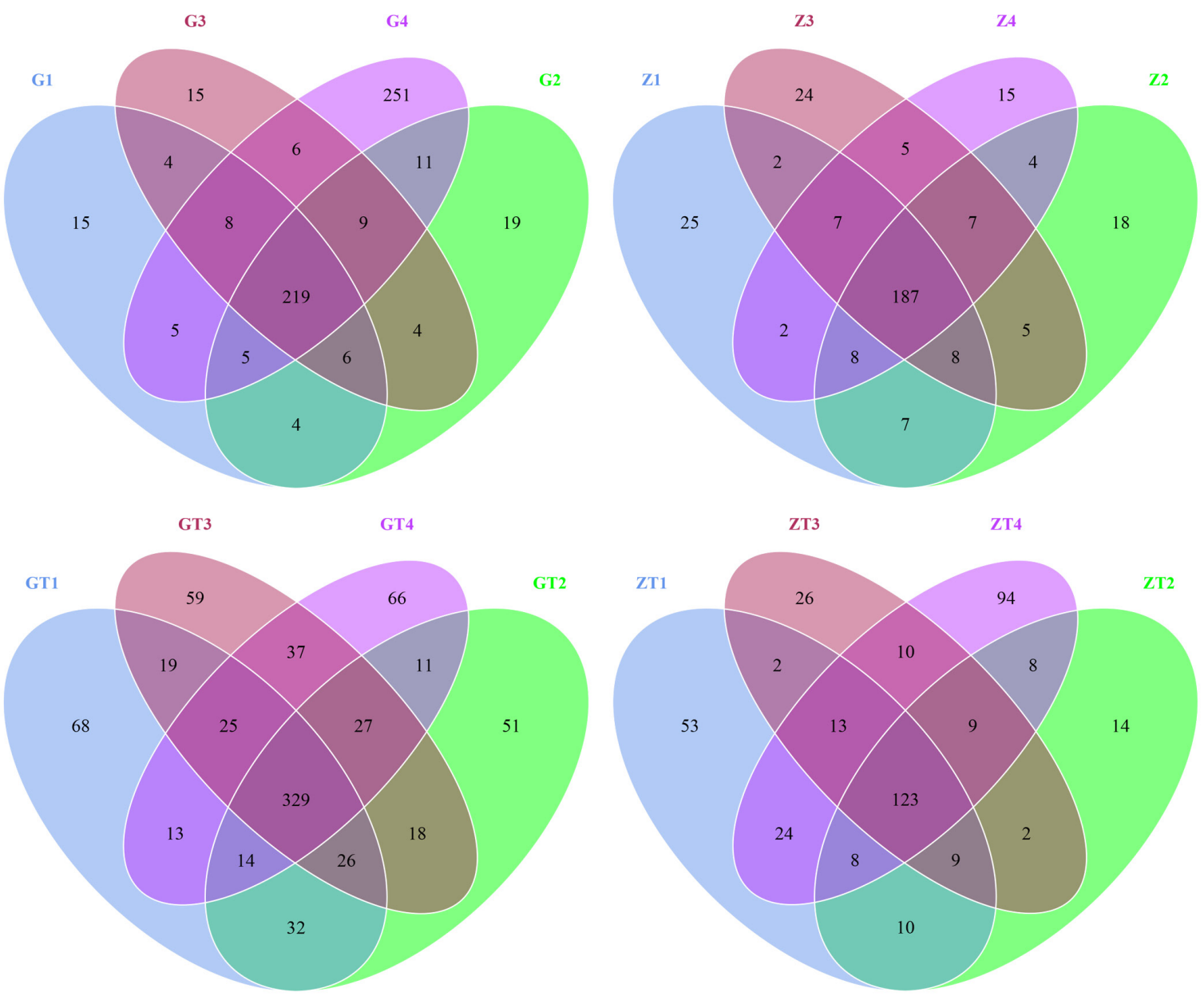

Figure 2: Scalar-Venn representation of shared genera among microbiomes associated with 4 sites in two patients before and after treatment. G1-4 and Z1-4 meaned the samples from patient $\mathrm{G}$ and patient Z before treatment respectively. GT1-4and ZT1-4 meaned the samples from the patient G and patient Z after treatment (represent as GT and ZT) respectively. The overlap section represented the shared OTUs between different samples.
A
B
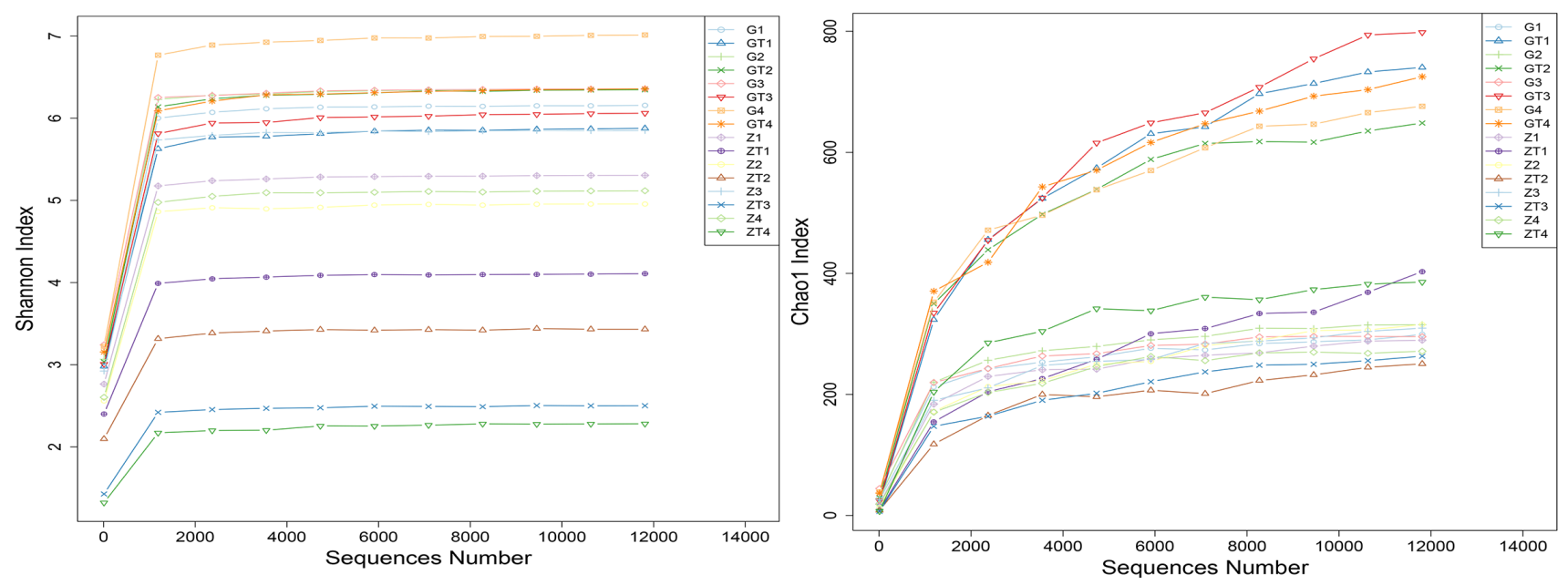

Figure 3: The rarefraction curves of Shannon index A. and Chaol index B. The horizontal axis represented sequence number, the vertical axis represented the shannon index in $\mathbf{A}$. and chaol index in $\mathbf{B}$. 
the Beta diversity index heatmap was generated (Figure 4C). The numerical values within the squares indicated the variation of coefficient between each two samples. 0 represents identity, 1 means totally different. The smaller the discrepancy coefficient was, the more similarity there was between two samples. Within one square, the upper and lower numerical values represented the weighted UniFrac and unweighted UniFrac distances respectively. Figure $4 \mathrm{C}$ showed that there was little difference between samples before treatment but more difference in microbial community of the same site after treatment. Moreover, after treatment, the microbial community shared less similarity between two patients (different groups). We also found whenever before or after treatment, the microbial similarity in the same site among the two patients had a less difference than that in one site before and after treatment.

\section{Variance analysis of species abundance}

All of the sequences were classified into 34 phyla. The top six most abundant bacteria in phylum level in each sample were shown in Table S4. The relative abundance of each phylum was indicated based on the homogenized OTU numbers (Figure 5). Before treatment,
A

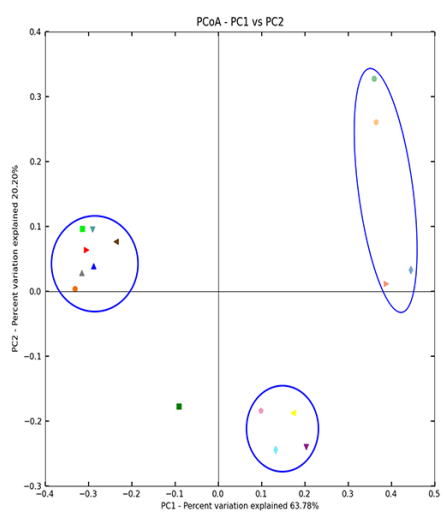

B
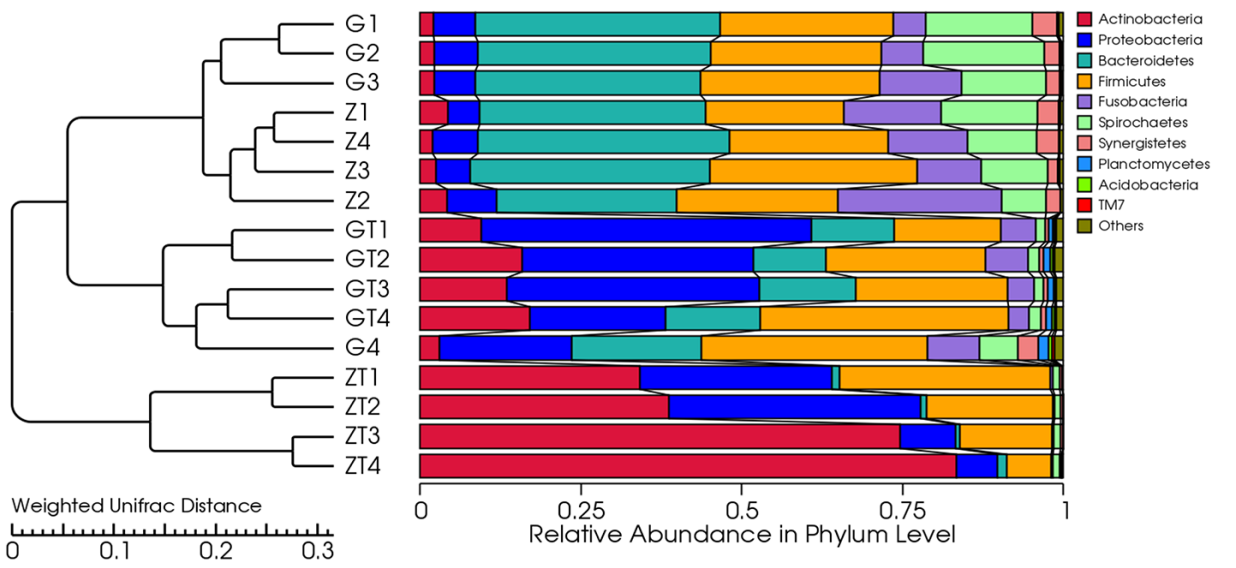

C

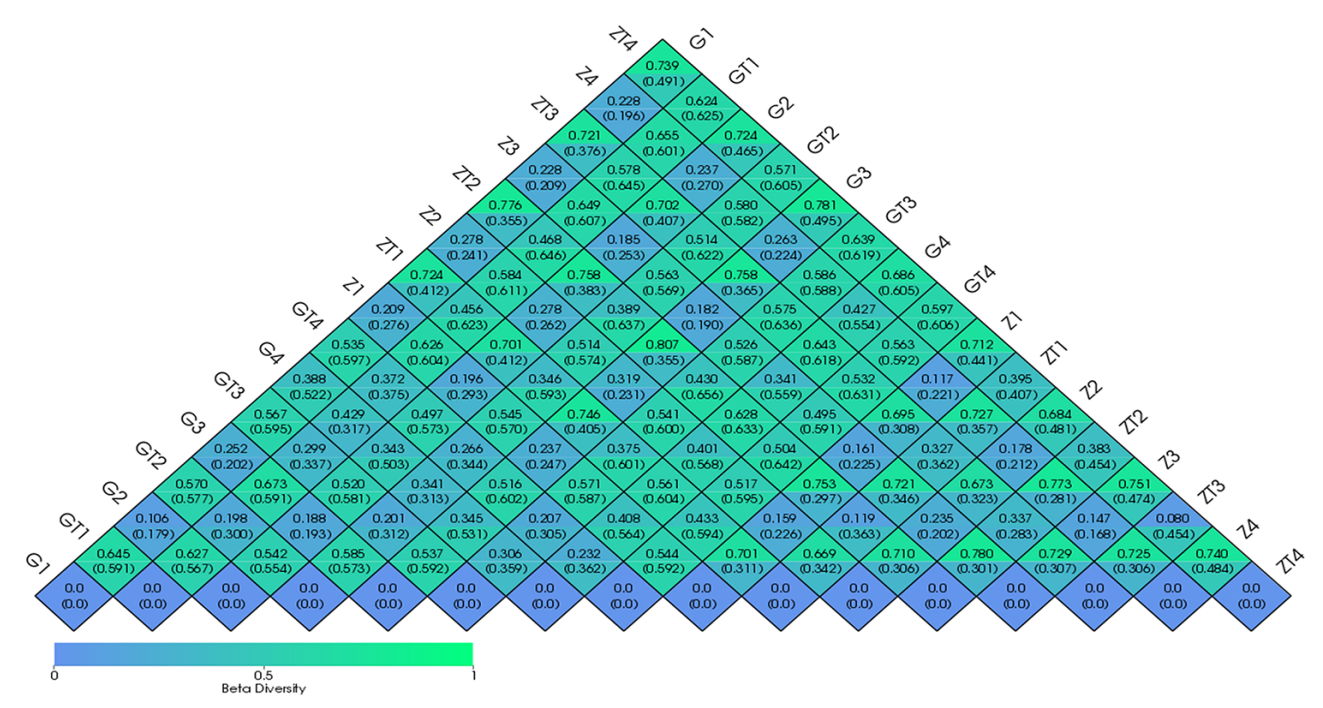

Figure 4: Beta-deversity analysis index. A. showed Principal coordinate analysis (PCoA) scores plot based in weighted UniFrac metrics. Each symbol represented each sample from patient G and Z before and after treatment. Principal components (PCs) 1 and 2 explained $63.78 \%$ and $20.20 \%$ of the variance respectively. B. showed the phylogenetic tree based on the Weighted UniFrac. The left part is the phylogenetic tree based on the similarity between samples; the right part is the heatmap of relative abundance in phylum level. $\mathbf{C}$. showed the $\beta$-diversity index heatmap.The numerical values within the squares indicated thecoefficient of difference between each two samples. The green color represented greater difference, and the blue color represented smaller difference. 
microbial community was dominated by the six most predominant bacteria: Bacteroidetes (20.2-39.2\%), Firmicutes (21.4-35.1\%), Spirochaetes ( 6-18.9\%), Proteobacteria (5-20.5\%), Fusobacteria (5.1-25.5\%) and Synergistetes (1.6-3.9\%), which was in accordance with previous studies $[4,5]$. The remaining genera contributed less than $4.9 \%$ in proportion. In contrast, the bacterial community after mechanical debridement exhibited a much different taxonomic composition, in which the most prevalent phyla became: Actinobacteria (9.5-83.4\%), Firmicutes (6.9-38.6\%), Proteobacteria (6.4-51.3\%), Bacteroidetes (0.8-15.1\%), Spirochaetes (1.0-1.9\%), Fusobacteria (0.2-6.7\%) (Table S4). Previous studies revealed a community dominated by the bacterial phyla Firmicutes, Actinobacteria, Bacteroidetes, Fusobacteria and Proteobacteriain in chronic periodontitis patients [12]. We found these five phyla also dominated the subgingival microbial community of GAgP patients. Moreover, Spirochaetes was also one of the predominant bacteria in these two aggressive periodontitis (AgP) patients which was reported previously [4, 5] [13]. Generally speaking, a shift from Gram-negative bacteria to Gram-positive bacteria was observed after treatment in our study. After periodontal therapy, the proportion of Actinobacteria and Proteobacteria increased dramatically while the proportion of Bacteroidetes, Spirochaetes, Fusobacteria decreased dramatically, which suggested these three anaerobic and Gram-negative bacteria Bacteroidetes, Spirochaetes, Fusobacteria were the putative periodontal pathogens for GAgP. Periodontal pathogens decreased after therapy with the increase of other bacteria, such as Actinobacteria and Proteobacteria. Actually, we knew that Porphyromonas gingivalis (Bacteroidetes), Treponemas denticola (Spirochaetes) and Fusobacterium nucleatum (Fusobacteria) are the main periodontal pathogens [9]. The most interesting phenomenon appeared when we analyzed the two patients separately. Before treatment, the six most predominant bacterial compositions of the two patients in phylum level were quite similar, though the distribution of each was slightly different. However, after treatment, the bacterial composition of the two patients exhibited a huge difference. For patients Z, Actinobacteria became the most dominant bacteria, especially in sites ZT3 and ZT4 (Actinobacteria accounts for 75 and 83\%) which suggested that the microbial transition after periodontal therapy might vary among different patients.

Based on the species abundance of each sample, we picked the top 35 species in genera level to generate a heatmap (Figure 6). The top 35 most abundant genera represented 57.2-91.35\% of the bacteria in each sample. Different colors represented different levels of relative abundance. As shown in Figure 6, before treatment, Sharpea, Moryella, Fusobacterium, Johnsonella, Peptococcus, Peptostreptococcus, Treponema, TG5,

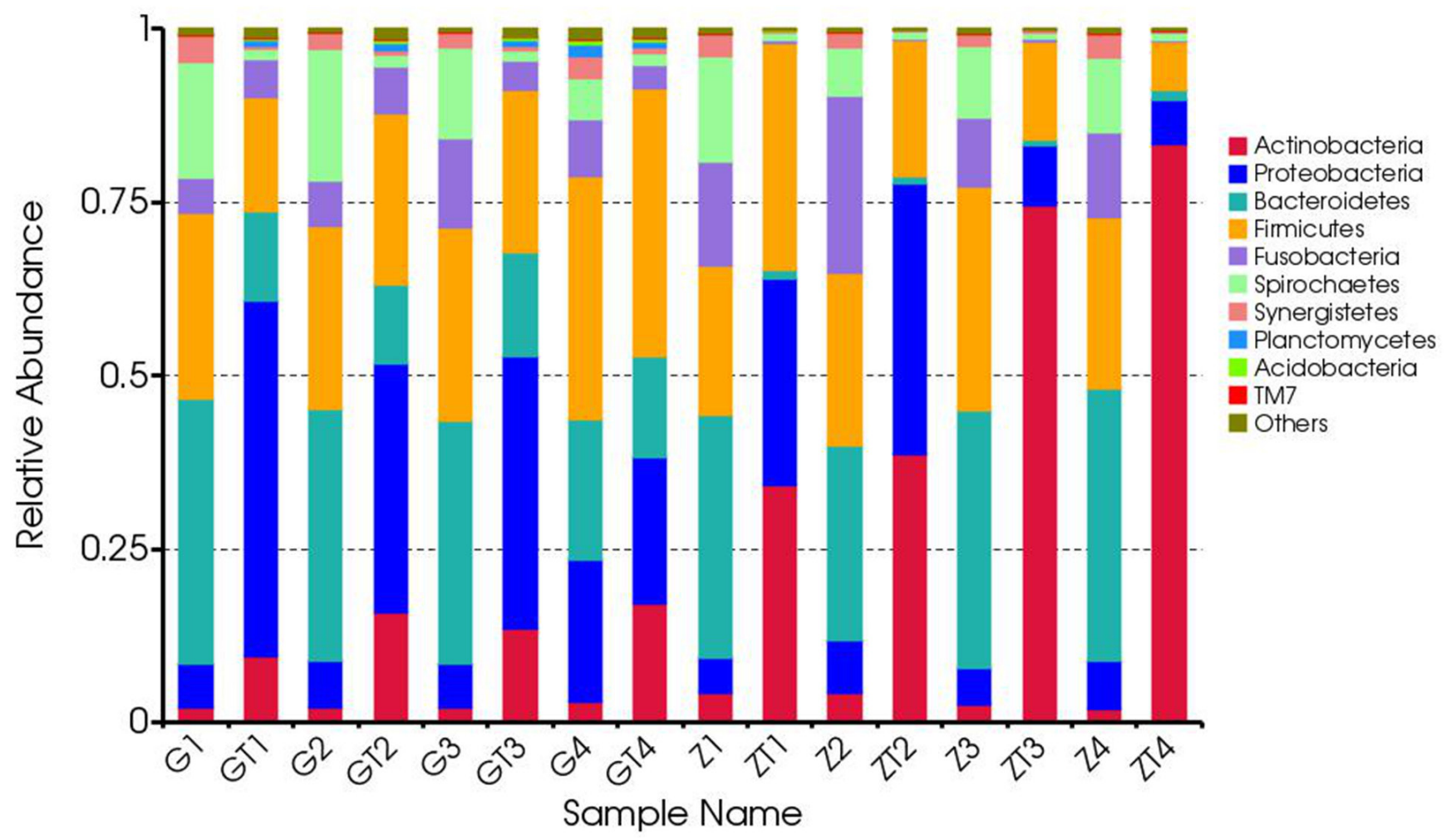

Figure 5: Composition and relative abundance communities based on 16S rDNA sequence in phylum level. The distribution of major phyla in the bacterial communities of samples before and after treatment. Sequences that could not be classified into any known group were labeled "other". 
Desulfobulbus, Filifactor, Tannerella, Porphyromonas, Megamonas, Escherichia, Selenomonas, Dialister, Megasphaera, Prevotella, Leptotrichia, Hylemonella, Campylobacter, Bacteroides, Syntrophomonas had a higher level of abundance. Then, after treatment, Kingella, Sphingopyxis, Lautropia, Capnocytophaga, Neisseria, Aggregatibacter, Corynebacterium, Actinomyces, Parascardovia, Veillonella, Rothia and Streptococcus had a higher level of abundance. We picked the most abundant phylum in each sample and annotated its belonging in phylum level (Table S5).

\section{DISCUSSION}

Many potential pathogens are associated with periodontitis, while traditional culture-dependent methods can not detect all oral microbiomes since most of them have a very little trace or are uncultivable. Metagenosome sequencing based on culture-independent methods can facilitate the study process of microbiome and their functions in the development of periodontitis. As a polymicrobial disease, there are many co-infecting species interacting with each other. The present study was designated to characterize the composition transition of subgingival microbiomes associated with GAgP patients pre- and post-treatment. While only two

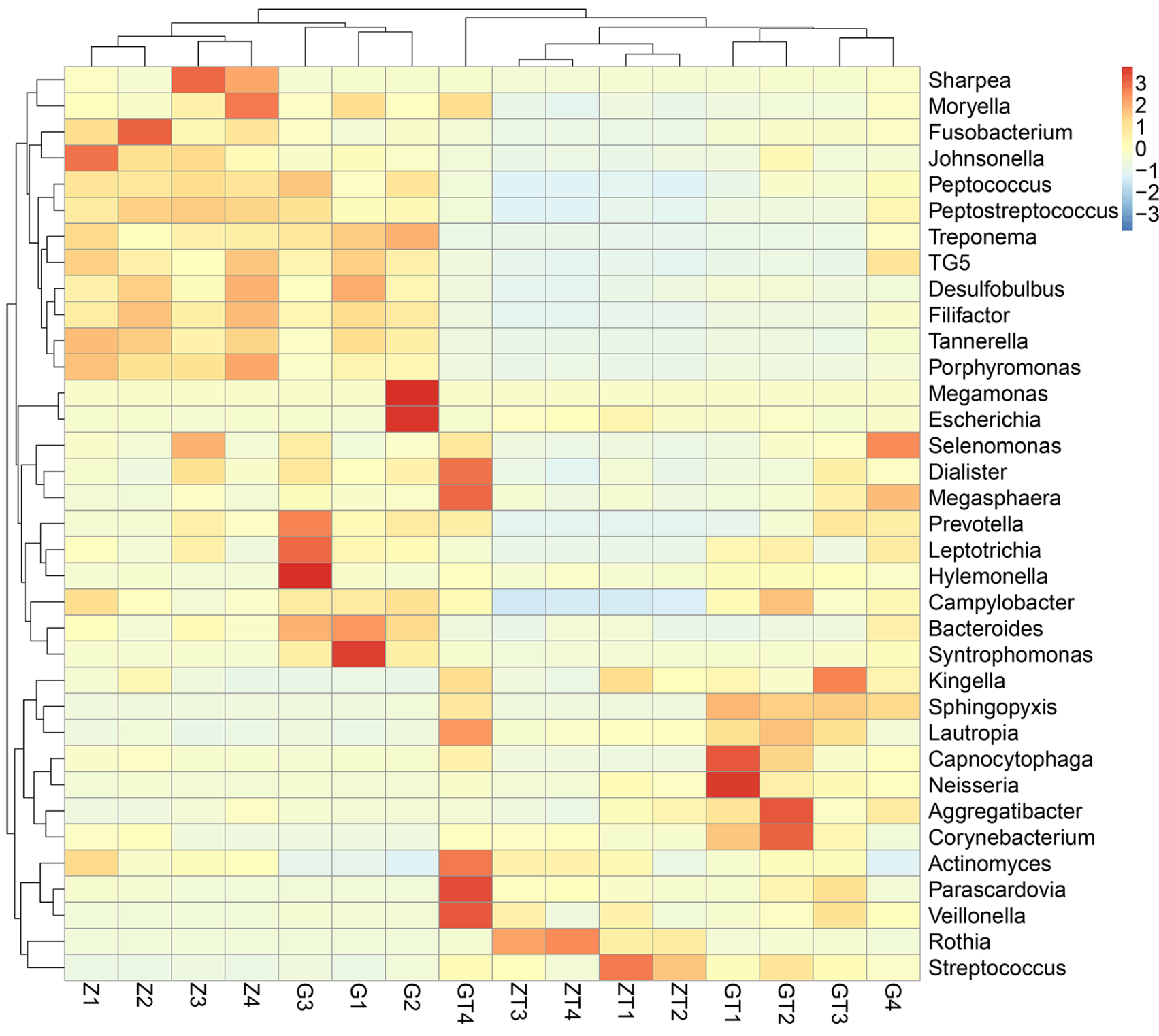

Figure 6: Relative abundance of the top 35 most prevalent genera in subgingival plaque samples before and after intervention. Only genera with more than $1 \%$ abundance were included. Each Bar displayed the normalized relative abundance, colors reflected relative abundance from low (blue) to high (red). 
subjects were involved (with each subject contributing one pooled sample from four periodontal sites pre- and post-treatment), the data was informative and provided a guideline for future studies. We have achieved sufficient sequencing depth to account for any bacterial species that constituted $0.02 \%$ or greater of the subgingival microbiota. Given that the sample size for each patient was small, we mainly focused on the comparison of subgingival plaque composition before and after non-surgical periodontal treatment, which might throw light on the study of the interaction between different oral microbiomes associated with periodontitis.

Many studies have identified that Aggregatibacter. actinomycetemcomitans, Porphyromonas. gingivalis, Tannerella. forsythia and Treponema. denticola were the predominant pathogens of GAgP $[4,5]$. In our study, we found that Bacteroidetes, Firmicutes, Spirochaetes, Proteobacteria, Fusobacteria and Synergistetes were the most 6 relatively abundant phylum before treatment. Studied have characterized that Aggregatibacter. actinomycetemcomitans belongs to Proteobacteria, Porphyromonas. gingivalis and Tannerella. forsythia belong to Bacteroidetes, and Treponema. denticola belongs to Spirochaetes in phylum level. Our study was consistent with all of these previous findings. Among these phyla, Spirochaetes also has been reported as a dominant bacterium in dental calculus [14]. Since calculus was a predisposing local factor, Spirochaetes in dental calculus might be a potential factor leading periodontitis. After treatment, the proportion of Actinobacteria and Proteobacteria increased dramatically while the proportion of Bacteroidetes, Spirochaetes and Fusobacteria decreased greatly. This phenomenon demonstrated that most of the main pathogens had been removed. We also found that the distribution of microbiomes after treatment between the two patients was different, which suggested that the commensal or normal microbiomes were diverse in different individuals. We suspected that change of physicochemical environments and their influence to each other might alter the distribution of the microbioms since their physicochemical characteristics were very different.

In this study, the subgingival microbiota was high in species richness but dominated by a few species or phylotypes, with significant shifts of microbiota that occurred after treatment. Also, the composition of the subgingival microbiota was highly individualized. The observed species richness in the subgingival microbiota was in general agreement with other studies [2, 4, 5].

Pre- and post-treatment samples showed significant quantitative and distributive species changes. Quantitatively, many known pathogenic species went from easily detectable to zero counts while the opposite occurred for many known health-associated bacteria. In most cases, the abundance of these known pathogens decreased dramatically, with some species, such as Fusobacterium, Treponema, Tannerella, Porphyromonas,
Prevotella, Campylobacter and Bacteroides. Conversely, known commensal such as Actinomyces, Veillonella and Rothia increased after treatment. Two suspected pathogens, Aggregatibacter and Capnocytophaga increased after treatment. The assignments of bacterial species to either pathogen or commensal have not always been consistent in the literature. As the clinical parameters improved after treatment, perhaps these species may be considered more beneficial than pathogenic. Some opportunistic pathogen increased after treatment, which can not cause diseases in normal conditions.

When comparing pre- and post-treatment samples, we observed a shift in the composition of the oral microbiota, supporting the well characterized transition from a Gram-negative dominated community in pre-treatment samples, to a Gram-positive dominated community in post-treatment samples. Not surprisingly, Gram-negative genera Fusobacterium, Treponema, Tannerella, Porphyromonas, Prevotella, Campylobacter and Bacteroides were significantly enriched in samples before treatment. Among these, three genera, Treponema, Tannerella, Porphyromonas, were of particular interest, as they include the species Porphyromonas gignivalis, Treponema denticola and Tannerella forsythia, proposed to form the pathogenic "red complexes" consortium in periodontitis [15]. Furthermore, the genus Prevotella also includes several known periodontal associated pathogens (Preveotell nigrescens, Preveotella intermedia and Prevotella melaninogenica) [16]. For post-treatment samples, Gram-negative genera Fusobacterium, Treponema, Tannerella, Porphyromonas, Prevotella, Campylobacter and Bacteroides were remarkably suppressed. Furthermore, a set of genera Kingella, Sphingopyxis, Lautropia, Capnocytophaga, Neisseria, Aggregatibacter, Corynebacterium, Actinomyces, Parascardovia, Veillonella, Rothia and Streptococcus increased abundance in the samples after treatment. Of them, Streptococcus, Actinomyces and Rothia were known as early colonizer species [17]. Surprisingly, both Aggregatibacter and Capnocytophaga were found to be more abundant after treatment at least in one sample, and one species of Aggregatibacter genus was Aggregatibacter actinomycetemcomitans, long been considered as a major etiologic agent of GAgP [18]. Since taxonomic resolution down to species-level was impossible to visualize, we were not able to determine if the sequencing reads counting for the genus Aggregatibacter is Aggregatibacter. actinomycetemcomitans, Aggregatibacter. aphrophilus or Aggregatibacter. segnis. Aggregatibacter contains the aforementioned three species which was previously implicated as the periodontal pathogen [19]. This phenomenon needs further investigation.

The findings in the present study might provide a novel framework to understand the pathogens and their mechanism of the GAgP from the gene level. However, the size of the samples was limited, more samples were 
needed to study the interaction between pathogens more deeply and find the functional genes of oral microbiomes.

\section{MATERIALS AND METHODS}

\section{Study subjects}

The study was approved by the Medical Ethical Committee of School of Stomatology, Shandong University (Protocol Number: 201302070). Two subjects (designated as $\mathrm{G}$ and $\mathrm{Z}$ ) with $\mathrm{GAgP}$ were recruited from Department of Periodontology, School of Stomatology, Shandong University. Both patients signed an informed consent prior to their enrollment in the study. A diagnosis of GAgP was determined based on the American Academy of Periodontology of Periodontal parameters [8]. Both of the subjects are non-smoking females (27 and 29 years old), who were free of systemic diseases and had not taken antibiotics within the past year for any reason, nor had they received any non-surgical or surgical periodontal therapy previously.

\section{Clinical examination and treatment}

An experienced dentist measured clinical periodontal parameters, delivered treatment and obtained subgingival plaques. Clinical parameters including PD and BOP on six sites per tooth and all teeth were recorded at baseline and one month after therapy. Both patients received full mouth scaling and root planning.

\section{Microbial sampling}

Subgingival plaque samples from mesio-buccal site of the first molar were obtained at baseline and at the four-week follow up so that a total of four samples before treatment and four samples after treatment for each patient were analyzed. Samples were taken from mesiobuccal site of four first molars with initial probing depths $\geq 5 \mathrm{~mm}$. Sampling sites were isolated with cotton rolls after all supragingival plaque and calculus were removed using sterile Gracey curettes and placed in $0.5 \mathrm{ml}$ PBS. After centrifuged at $3000 \mathrm{~g}$ for $5 \mathrm{~min}$, the supernatants were frozen at $-80^{\circ} \mathrm{C}$ for further processing.

\section{Extraction of genome DNA}

Total genome DNA from samples was extracted using CTAB method. DNA concentration and purity was monitored on $2 \%$ agarose gel. According to the concentration, DNA was diluted to $1 \mathrm{ng} / \mu \mathrm{l}$ using sterile water.

\section{Bacterial 16s rDNA gene amplification and Illumina Sequencing}

We picked the fragments containing V4 16s rDNA hypervariable region for gene amplification [20,21]. The sequencing method was described by Caporaso et al [22]. The primer set selected for amplifying the V4 16s rDNA hypervariable region was $515 \mathrm{f} / 806 \mathrm{r}$. It exhibited few biases and yielded accurate phylogenetic and taxonomic information. The reverse primer contained a 6-bp barcode which was unique to each sample. All PCR reactions were carried out in $30 \mu \mathrm{l}$ reaction system with $15 \mu$ l of Phusion ${ }^{\circledR}$ High-Fidelity PCR Master Mix (New England Biolabs), $0.2 \mu \mathrm{mol} \mathrm{l}^{-1}$ of forward and reverse primers, and about 10ng template DNA. Thermal cycling consisted of initial denaturation at $98^{\circ} \mathrm{C}$ for $1 \mathrm{~min}$, followed by 30 cycles of denaturation at $98^{\circ} \mathrm{C}$ for $10 \mathrm{~s}$, annealing at $50^{\circ} \mathrm{C}$ for $30 \mathrm{~s}$, and elongation at $72^{\circ} \mathrm{C}$ for $60 \mathrm{~s}$ and finally $72^{\circ} \mathrm{C}$ for $5 \mathrm{~min}$. The quantification and qualification of PCR products were carried on by operating electrophoresis on $2 \%$ agarose gel. PCR products were mixed in equidensity ratios. Then, mixed PCR products were purified with V4 hypervariable region was picked for PCR amplification of subgingival samples GeneJET Gel Extraction Kit (Thermo Scientific). Finally, the library was sequenced by Illumina MiSeq platform and 250bp/300bp paired-end reads were generated.

\section{Data analysis}

Paired-end reads from the original DNA fragments were merged by FLASH V1.2.7, http://ccb.jhu.edu/ software/FLASH/ [23] -a very fast and accurate analysis tool which was designed to merge paired-end reads when there were overlaps between two reads (reads 1 and reads 2). After strict quality-filtering [24], paired-end reads were assigned to each sample according to the unique barcodes. Sequences were analyzed using QIIME software package V1.7.0, http://qiime.org/scripts/split_libraries_fastq.html. First, reads were filtered by QIIME quality filters. The process involved four sections:1) min per_read length: minimum number of consecutive high-quality base calls to retain read (as percentage of total read length). 2) max_bad_run_length: maximum number of consecutive low quality base calls allowed before truncating a read. 3) sequence_max_n: maximum number of ambiguous (N) characters allowed in a sequence. 4) phred_quality score: last quality score considered low quality [25]. Then we used UPARSE pepline V7.0, 1001, http:// drive5.com/uparse/ [26] to pick operational taxonomic units (OTUs) by making OTU table. Sequences with $\geq 97 \%$ similarity were assigned to the same OTUs. We picked a representative sequences for each OTU and used Ribosomal Database Project (RDP) classifier V2.2, http://sourceforge.net/projects/rdp-classifier/ [27] and 
Greengenes database http:/greengenes.lbl.gov/cgi-bin/ nph-index.cgi [28] to annotate taxonomic information for each representative sequence. We used MUSCLE V3.8.31, http://www.drive5.com/muscle/ [29, 30] software to get the phylogenetic relationship based on the respective OTUs.

In order to compute Alpha diversity, we rarified the OTU table and calculate three metrics by QIIME: Shannon index estimates the diversity and evenness; Observed Species estimates the amount of unique OTUs found in each sample, and Chaol estimates the richness. Rarefaction curves were generated based on these three metrics. At the same time, we calculated the Simpson's Index to visualize evenness.

QIIME calculates both weighted and unweighted unifrac [31-33], which are phylogenetic measures of beta diversity. We used weighted unifrac for Principal Coordinate Analysis (PCoA) and Unweighted Pair Group Method with Arithmetic mean (UPGMA). PCoA helps to get principal coordinates and visualize them from complex, multidimensional data. It takes a transformation from a distance matrix to a new set of orthogonal axes. By which the maximum variation factor is demonstrated by first principal coordinate, and the second maximum one by the second principal coordinate, and so on. UPGMA is a type of hierarchical clustering method using average linkage and can be used to interpret the distance matrix. A schematic overview of the experimental work-flow and applied bioinformatic procedure was given in Figure S1.

\section{ACKNOWLEDGMENTS}

This study was funded by the National Natural Science Foundation of China (No. 81670993, 81371157), Science and Technology Program of Shandong Province (No. 2014GSF118075) and the Construction Engineering Special Fund of Taishan Scholars (No. ts201511106). The funders had no role in study design, data collection and analysis, decision to publish, or preparation of the manuscript.

\section{CONFLICTS OF INTEREST}

The authors declare that they have no conflict of interest declared.

\section{REFERENCES}

1 Bik EM, Long CD, Armitage GC, Loomer P, Emerson J, Mongodin EF, Nelson KE, Gill SR, Fraser-Liggett CM and Relman DA. Bacterial diversity in the oral cavity of 10 healthy individuals. ISME J. 2010; 4:962-974.

2 Belda-Ferre P, Alcaraz LD, Cabrera-Rubio R, Romero H, Simon-Soro A, Pignatelli M and Mira A. The oral metagenome in health and disease. ISME J. 2012; 6:46-56.
3 Kroes I, Lepp PW and Relman DA. Bacterial diversity within the human subgingival crevice. Proc Natl Acad Sci U S A. 1999; 96:14547-14552.

4 Paster BJ, Boches SK, Galvin JL, Ericson RE, Lau CN, Levanos VA, Sahasrabudhe A and Dewhirst FE. Bacterial diversity in human subgingival plaque. J BACTERIOL. 2001; 183:3770-3783.

5 Dewhirst FE, Chen T, Izard J, Paster BJ, Tanner AC, Yu WH, Lakshmanan A and Wade WG. The human oral microbiome. J BACTERIOL. 2010; 192:5002-5017.

6 Faveri M, Mayer MP, Feres M, de Figueiredo LC, Dewhirst FE and Paster BJ. Microbiological diversity of generalized aggressive periodontitis by $16 \mathrm{~S}$ rRNA clonal analysis. Oral Microbiol Immunol. 2008; 23:112-118.

7 Paster BJ, Olsen I, Aas JA and Dewhirst FE. The breadth of bacterial diversity in the human periodontal pocket and other oral sites. PERIODONTOL 2000. 2006; 42:80-87.

81999 International International Workshop for a Classification of Periodontal Diseases and Conditions. Papers. Oak Brook, Illinois, October 30-November 2, 1999. Ann Periodontol. 1999; 4:1-112.

9 Ranney RR. Classification of periodontal diseases. PERIODONTOL 2000. 1993; 2:13-25.

10 Feng XH, Zhang L, Meng HX, Xu L, Chen ZB and Shi D. [Prevalence of putative periodontal microorganisms in Chinese patients with aggressive periodontitis]. Zhonghua Kou Qiang Yi Xue Za Zhi. 2006; 41:344-347.

11 Peiffer JA, Spor A, Koren O, Jin Z, Tringe SG, Dangl JL, Buckler ES and Ley RE. Diversity and heritability of the maize rhizosphere microbiome under field conditions. Proc Natl Acad Sci U S A. 2013; 110:6548-6553.

12 Wang J, Qi J, Zhao H, He S, Zhang Y, Wei S and Zhao F. Metagenomic sequencing reveals microbiota and its functional potential associated with periodontal disease. Sci Rep. 2013; 3:1843.

13 Oliveira RR, Fermiano D, Feres M, Figueiredo LC, Teles FR, Soares GM and Faveri M. Levels of Candidate Periodontal Pathogens in Subgingival Biofilm. J DENT RES. 2016; 95:711-718.

14 Gupta S, Jain PK, Kumra M, Rehani S, Mathias Y, Gupta $\mathrm{R}$, Mehendiratta $\mathrm{M}$ and Chander A. Bacterial Viability within Dental Calculus: An Untrodden, Inquisitive ClinicoPatho- Microbiological Research. J Clin Diagn Res. 2016; 10:C71-C75.

15 Socransky SS, Haffajee AD, Cugini MA, Smith C and Kent RJ. Microbial complexes in subgingival plaque. J CLIN PERIODONTOL. 1998; 25:134-144.

16 Socransky SS and Haffajee AD. Periodontal microbial ecology. PERIODONTOL 2000. 2005; 38:135-187.

17 Kolenbrander PE, Palmer RJ, Rickard AH, Jakubovics NS, Chalmers NI and Diaz PI. Bacterial interactions and successions during plaque development. PERIODONTOL 2000. 2006; 42:47-79. 
18 Koenoenen E and Mueller H. Microbiology of aggressive periodontitis. PERIODONTOL 2000. 2014; 65:46-78.

19 Petrukhina NB, Zorina OA, Shikh EV, Shibaeva AV and Shevelev AB. [Study of mutual dependence of periodontal and colonic microbiome in health and pathology using NSG-sequencing]. Stomatologiia. 2016; 95:8-13.

20 Kelly JJ, Minalt N, Culotti A, Pryor M and Packman A. Temporal variations in the abundance and composition of biofilm communities colonizing drinking water distribution pipes. PLOS ONE. 2014; 9:e98542.

21 Wagner MR, Lundberg DS, Coleman-Derr D, Tringe SG, Dangl JL and Mitchell-Olds T. Natural soil microbes alter flowering phenology and the intensity of selection on flowering time in a wild Arabidopsis relative. ECOL LETT. 2014; 17:717-726.

22 Caporaso JG, Lauber CL, Walters WA, Berg-Lyons D, Lozupone CA, Turnbaugh PJ, Fierer N and Knight R. Global patterns of $16 \mathrm{~S}$ rRNA diversity at a depth of millions of sequences per sample. Proc Natl Acad Sci U S A. 2011; 108 Suppl 1:4516-4522.

23 Magoc $\mathrm{T}$ and Salzberg SL. FLASH: fast length adjustment of short reads to improve genome assemblies. BIOINFORMATICS. 2011; 27:2957-2963.

24 Bokulich NA, Subramanian S, Faith JJ, Gevers D, Gordon J, Knight R, Mills DA and Caporaso JG. Quality-filtering vastly improves diversity estimates from Illumina amplicon sequencing. NAT METHODS. 2013; 10:57-59.

25 Caporaso JG, Kuczynski J, Stombaugh J, Bittinger K, Bushman FD, Costello EK, Fierer N, Pena AG, Goodrich JK, Gordon JI, Huttley GA, Kelley ST, Knights D, et al. QIIME allows analysis of high-throughput community sequencing data. NAT METHODS. 2010; 7:335-336.
26 Edgar RC. UPARSE: highly accurate OTU sequences from microbial amplicon reads. NAT METHODS. 2013; 10:996998.

27 Cole JR, Wang Q, Cardenas E, Fish J, Chai B, Farris RJ, Kulam-Syed-Mohideen AS, McGarrell DM, Marsh T, Garrity GM and Tiedje JM. The Ribosomal Database Project: improved alignments and new tools for rRNA analysis. NUCLEIC ACIDS RES. 2009; 37:D141-D145.

28 DeSantis TZ, Hugenholtz P, Larsen N, Rojas M, Brodie EL, Keller K, Huber T, Dalevi D, Hu P and Andersen GL. Greengenes, a chimera-checked 16S rRNA gene database and workbench compatible with ARB. Appl Environ Microbiol. 2006; 72:5069-5072.

29 Edgar RC. MUSCLE: a multiple sequence alignment method with reduced time and space complexity. BMC BIOINFORMATICS. 2004; 5:113.

30 Edgar RC. MUSCLE: multiple sequence alignment with high accuracy and high throughput. NUCLEIC ACIDS RES. 2004; 32:1792-1797.

31 Lozupone $\mathrm{C}$ and Knight R. UniFrac: a new phylogenetic method for comparing microbial communities. Appl Environ Microbiol. 2005; 71:8228-8235.

32 Lozupone C, Hamady M and Knight R. UniFrac - an online tool for comparing microbial community diversity in a phylogenetic context. BMC BIOINFORMATICS. 2006; 7:371.

33 Lozupone C, Lladser ME, Knights D, Stombaugh J and Knight R. UniFrac: an effective distance metric for microbial community comparison. ISME J. 2011; 5:169172. 